Address correspondence to: J. Andrew Alspaugh, Duke University Medical Center, Division of Infectious Diseases and International Health, 1543 Busse Building, Department of Medicine, Durham, North Carolina 27710, USA. Phone: 919.684.0045; Fax: 919.684.8902; E-mail: andrew.alspaugh@duke.edu.

1. Saegeman V, Maertens J, Meersseman W, Spriet I, Verbeken E, Lagrou K. Increasing incidence of mucormycosis in University Hospital, Belgium. Emerg Infect Dis. 2010;16(9):1456-1458.

2. Bitar D, et al. Increasing incidence of zygomycosis (mucormycosis), France, 1997-2006. Emerg Infect Dis. 2009;15(9):1395-1401.

3. Marty FM, Cosimi LA, Baden LR. Breakthrough zygomycosis after voriconazole treatment in recipients of hematopoietic stem-cell transplants. N Engl J Med. 2004;350(9):950-952.

4. Kontoyiannis DP, et al. Zygomycosis in a tertiary-care cancer center in the era of Aspergillus-active antifungal therapy: a case-control observational study of 27 recent cases. J Infect Dis. 2005; 191(8):1350-1360

5. Alspaugh JA, Perfect JR. Infections due to zygomycetes and other rare fungal opportunists. Semin Respir Crit Care Med. 1997;18(3):265-279.

6. Roden MM, et al. Epidemiology and outcome of zygomycosis: a review of 929 reported cases. Clin Infect Dis. 2005;41(5):634-653.

7. Gebremariam T, et al. CotH3 mediates fungal invasion of host cells during mucormycosis. J Clin Invest. 2014;124(1):237-250.

8. Frater JL, Hall GS, Procop GW. Histologic features of zygomycosis: emphasis on perineural invasion and fungal morphology. Arch Pathol Lab Med.
2001;125(3):375-378

9. Liu M, et al. The endothelial cell receptor GRP78 is required for mucormycosis pathogenesis in diabetic mice. J Clin Invest. 2010;120(6):1914-1924.

10. Schmidt CS, et al. NDV-3, a recombinant alum-adjuvanted vaccine for Candida and Staphylococcus aureus, is safe and immunogenic in healthy adults. Vaccine. 2012;30(52):7594-7600.

11. Crameri R, Blaser K. Allergy and immunity to fungal infections and colonization. Eur Respir J. 2002; 19(1):151-157.

12. Gravelat FN, et al. Aspergillus galactosaminogalactan mediates adherence to host constituents and conceals hyphal beta-glucan from the immune system. PLoS Pathog. 2013;9(8):e1003575.

13. O'Meara TR, Holmer SM, Selvig K, Dietrich F, Alspaugh JA. Cryptococcus neoformans Rim 101 is associated with cell wall remodeling and evasion of the host immune responses. MBio. 2013; 4(1):e00522-12.

\title{
More than skin deep: connecting melanocyte pigmentation and angiogenic diseases
}

\author{
Christopher D. Kontos
}

\author{
Department of Medicine, Division of Cardiology, Department of Pharmacology and Cancer Biology, \\ Duke University Medical Center, Durham, North Carolina, USA.
}

\begin{abstract}
Epidemiological studies have identified racial differences in susceptibility to numerous diseases, including several ocular and skin diseases characterized by increased vascular growth. In most cases, the specific mechanisms and genetic variants responsible for these differences have remained elusive. In this issue of the JCI, Adini et al. explore a direct connection between skin pigmentation and susceptibility to angiogenic diseases and identify an extracellular matrix protein that is regulated by melanogenesis and potently modulates angiogenesis.
\end{abstract}

\section{Skin pigmentation and angiogenesis dysfunction}

An important and ongoing challenge in the postgenomic era is the successful identification of gene variants that render certain individuals susceptible to specific diseases. For example, it is well known that individuals with less skin pigmentation have a higher incidence of angiogenic ocular and skin disease compared with darker-skinned individuals. Genetic variation drives differences in pigmentation; however, a genetic link between skin color and angiogenic disease has not been identified. Studies on ocular diseases indicate that individuals of European descent are twice as likely to develop age-related macular degeneration (AMD) as African-Ameri-

Conflict of interest: The author has declared that no conflict of interest exists.

Citation for this article: J Clin Invest. 2014;

124(1):76-79. doi:10.1172/JCI73559. cans or Asian-Americans (1). Furthermore, ocular melanoma is almost 20 times more common in light-skinned individuals (2), and several skin diseases, including melanoma and hemangioma, are more common in those of European descent $(3,4)$. The fact that these diseases are all characterized by increased vascular growth suggests a potential unexplored link between skin pigmentation and angiogenesis.

In this issue of the JCI, Adini and colleagues investigate the connection between skin color and disease susceptibility (5). The authors hypothesized that pigmented cells from light- or darkskinned individuals differentially express a proangiogenic factor. They observed that angiogenesis in the cornea and in healing wounds is considerably increased in C57-albino mice, which have decreased melanin synthesis due to a mutation in the tyrosinase gene, compared with their pigmented counterparts (C57BL/6 mice).
Using a cell-culture model of sprout formation, Adini et al. performed a detailed assessment of the effects of melanocyte-conditioned media on EC migration. Conditioned media were prepared by culturing melanocytes from albino and pigmented mice and, in a complementary fashion, from European- and African-Americans. The authors demonstrated that nonpigmented melanocytes secrete a factor that promotes microvascular EC migration and proliferation in vitro. These data are consistent with a melanocyte-secreted factor playing a role in promoting blood vessel growth.

\section{Fibromodulin: a potent angiogenic factor}

In order to identify the specific factor or factors responsible for the observed effects, the authors performed a targeted microarray that compared expression of genes predicted to encode secreted proteins in pigmented and nonpigmented melanocytes (5). Fibromodulin (FMOD), an ECM protein of the small leucine-rich proteoglycan (SLRP) family, was expressed to a much greater degree in nonpigmented melanocytes both in vitro and in vivo. Silencing or antibody blocking of FMOD substantially inhibited the angiogenic effects of conditioned media from nonpigmented mela- 

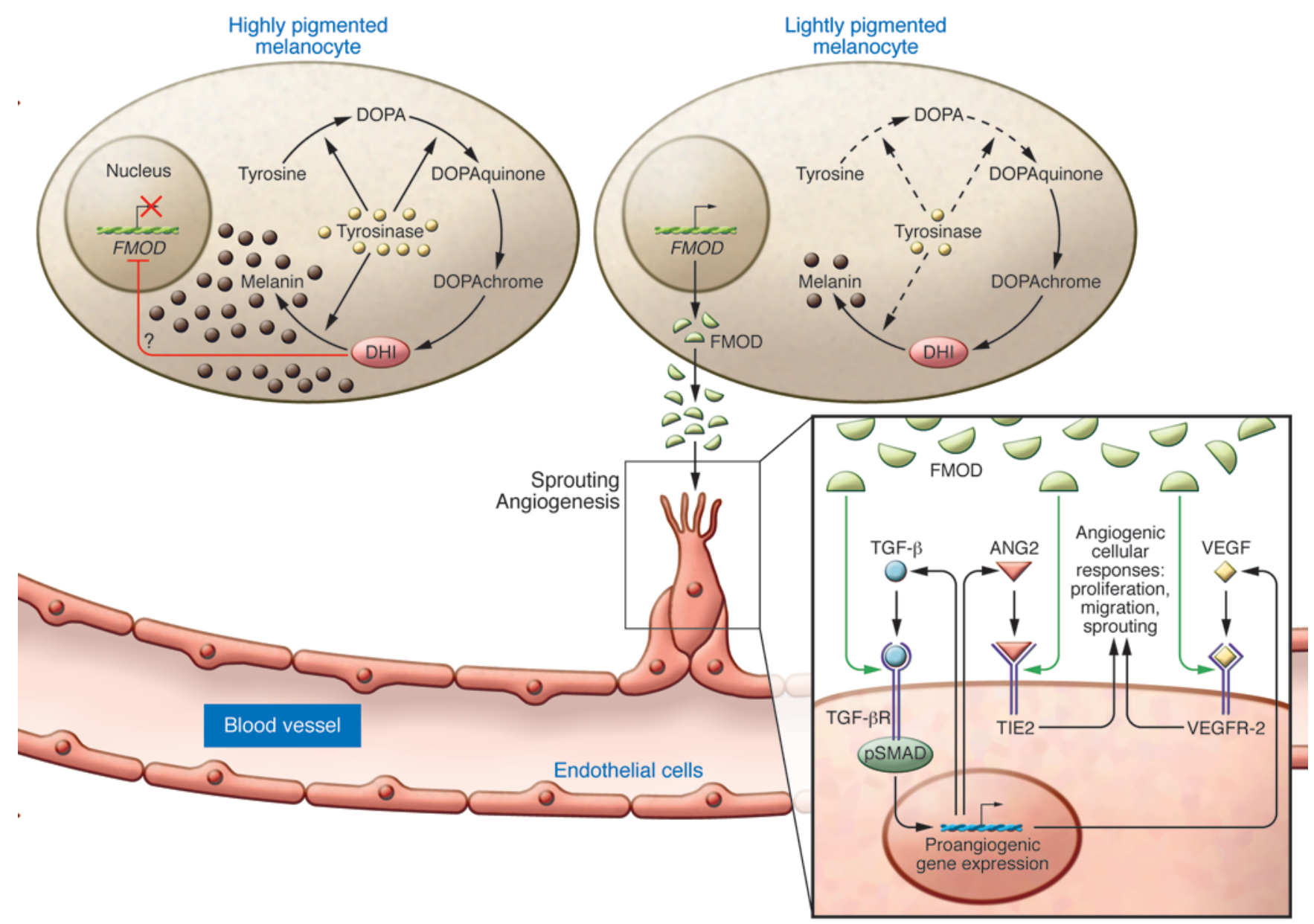

Figure 1

Potential mechanisms by which nonpigmented melanocytes might promote FMOD-mediated angiogenesis. Highly pigmented melanocytes have greater expression and activity of the enzyme tyrosinase, which regulates multiple steps in melanogenesis. The melanogenesis intermediate DHI inhibits FMOD expression through as-yet-unknown mechanisms, and the increased tyrosinase activity in heavily pigmented cells corresponds with reduced expression of $F M O D$ mRNA and protein, although melanin does not directly regulate FMOD expression. In lightly pigmented cells, the lack of tyrosinase activity enhances the production and secretion of FMOD from melanocytes, which promotes angiogenic cellular responses of nearby endothelial cells. At the level of the endothelial cell (inset), FMOD promotes activation of TGF- $\beta$ receptor signaling (through SMAD phosphorylation) and upregulation of angiogenic growth factors, including VEGF and ANG2, while repressing the vascular stabilizing factor ANG1 (not shown) through unknown mechanisms. VEGF and ANG2 activate their cognate endothelial receptor tyrosine kinases, VEGFR-2 and TIE2, to induce angiogenic cellular responses.

nocytes. Moreover, recombinant FMOD rendered pigmented melanocytes angiogenic. Strikingly, the addition of FMOD alone was as effective as the addition of the potent angiogenic factors VEGF and bFGF at promoting EC sprouting in vitro and vascular growth in vivo.

The role of FMOD and related SLRPs, including decorin and biglycan, has been explored previously under a variety of inflammatory and angiogenic conditions (6-9). Somewhat surprisingly, unlike decorin-null mice, mice lacking FMOD displayed no defects in corneal angiogenesis (7); however, the work by Adini et al. reveals a likely explanation for this appar- ently negative result (5). The original Fmod knockout was generated in a black (i.e., pigmented) mouse background, which would already express low baseline levels of FMOD; therefore, these mice should not be drastically affected by loss of FMOD. Adini et al. effectively proved this point by comparing vascular growth in pigmented or nonpigmented Fmod-null mice that were genetically identical except for a mutation in the tyrosinase gene of C57-albino mice. They found that endothelial cell infiltration into Matrigel plugs was indistinguishable between black wild-type mice and white Fmod-null mice, suggesting that FMOD is responsible for the differ- ence in angiogenesis. Moreover, the lack of FMOD in white mice noticeably decreased vascularity and reduced neovascularization in two models of ocular angiogenesis. Together, these results provide strong evidence that FMOD is at least partly responsible for the enhanced ocular and skin angiogenesis observed in light-skinned or nonpigmented individuals.

How does FMOD regulate angiogenesis? To begin to investigate FMOD's angiogenic regulatory effects, Adini et al. investigated a previously described interaction between FMOD and TGF- $\beta 1$ (8) and demonstrated that FMOD-induced EC migration could be blocked by pretreat- 
ment with an inhibitory TGF- $\beta$ antibody (5). Exogenous FMOD induced expression of TGF- $\beta$ RII, SMAD 1 , and SMAD 5 in ECs, implying an autocrine loop in which FMOD upregulates TGF- $\beta$ and its receptor, leading to activation of TGF- $\beta$ signaling and vascular growth (Figure 1). However, the requirement of TGF- $\beta$ signaling for the observed angiogenic effects of FMOD has not been tested. While the data from Adini et al. indicate that TGF- $\beta$ signaling is important (5), a paper published very recently suggests that additional mechanisms are likely at play (Figure 1). Jian et al. demonstrated that recombinant FMOD induces the adhesion, spreading, and tube-like formation (i.e., capillary morphogenesis) of ECs in vitro and promotes in vivo formation of new blood vessels in the chick chorioallantoic membrane assay (10). FMOD increased expression of angiopoietin 2 (ANG2) and VEGF, which are both potent angiogenic growth factors, and decreased expression of the vascular stabilizing factor ANG1. These results suggest that FMOD may modulate a broader range of vascular growth factors than just the TGF- $\beta$ pathway.

\section{Perspectives and future directions}

In fact, it is not unexpected that FMOD would have wide-reaching targets. ECM proteins have complex effects on angiogenesis and likely affect many angiogenic signaling pathways, including those mediated by receptor tyrosine kinases, integrins, and $G$ protein-coupled receptors (11). The SLRPs appear to affect multiple angiogenesis-associated pathways as well (6). Decorin, perhaps the best-studied SLRP, can bind directly and with high affinity to multiple receptors, including the EGF receptor, the hepatocyte growth factor receptor MET, and the IGF-I receptor. While decorin binding promotes transient activation and subsequent degradation of EGFR and MET $(12,13)$, it can also activate IGF-IR (14). The ability of SLRPs to bind and regulate multiple receptors raises the possibility that FMOD regulates angiogenesis through direct interactions with endothelial receptors, such as those for VEGF and the angiopoietins, which are critical regulators of vessel growth (15). Thus, to fully understand FMOD's mechanisms of action, its interaction with binding partners at the EC surface will require further exploration.

What are the upstream regulators of FMOD in melanocytes? Intriguingly, Adini et al. demonstrated that FMOD expression appears to be highly regulated by melanocyte pigmentation (5) (Figure 1). The removal of tyrosine, the critical building block in melanin synthesis, from the cellular growth medium enhanced FMOD expression, despite the persistence of melanin polymer within the cells. Furthermore, FMOD expression was reduced by the addition of 5,6-dihydroxyindole (DHI), an intermediate in eumelanin synthesis. Together, these results demonstrate that melanin itself does not directly regulate FMOD expression, but rather an intermediate melanin synthesis pathway metabolite is responsible. The identification of specific intermediates in melanogenesis that modulate FMOD expression will be critical for complete understanding of the contribution of FMOD to angiogenic diseases and may provide unique targets for disease treatment.

Individuals within racial groups have wide variations in skin color, leading to the question of whether genetic variants in other components of the melanin synthesis pathway confer differential susceptibility to angiogenic diseases via altered FMOD expression. In this regard, the results of Adini et al. (5) suggest that FMOD expression is an important biomarker of angiogenic disease susceptibility in the eyes, skin, and possibly other organs. For example, differential gene expression analysis revealed that FMOD is upregulated in glioblastoma, a highly malignant and angiogenic brain tumor, compared with the relatively benign pilocytic astrocytoma (16). This correlation raises the possibility that FMOD could be a marker of disease severity, at least in brain tumors. Conversely, a number of cardiovascular diseases are characterized by loss of vascular density (so-called vascular rarefaction), including diabetes mellitus and hypertension, both of which are more common in African-Americans than in those of European descent in the US. Based on these observations, it is interesting to speculate that reduced expression of FMOD in individuals with higher levels of pigmentation might indicate a predisposition toward insufficient vascular density. The SLRPs, including FMOD, have been studied extensively in inflammation and wound healing, and Fmodnull mice exhibit delayed wound closure (9). Although it is unclear whether angiogenesis deficiencies are involved in the reduced wound healing in these mice, loss of FMOD likely affects a variety of other tissues and cell types besides ECs. In this regard, it is conceivable that differential regulation of FMOD plays an important role in other nonvascular diseases; however, it remains to be determined whether this is a function of skin pigmentation.

While it is clear that the role of FMOD in angiogenic diseases is not fully understood, the results of Adini et al. (5) reveal an important and previously unrecognized component of genetic susceptibility to these conditions. Moreover, the ability to effectively inhibit angiogenesis in vivo with a blocking antibody points to FMOD as a potential therapeutic target in a variety of angiogenic disease states. Additional studies, particularly in humans across the spectrum of skin pigmentation, will ultimately be necessary to address the role of FMOD as both a biomarker and a therapeutic target in vascular diseases of the eyes, skin, and potentially other tissues.

Address correspondence to: Christopher D. Kontos, Department of Medicine, Division of Cardiology; Department of Pharmacology and Cancer Biology, Duke University Medical Center, Durham, North Carolina 27710, USA. Phone: 919.684.2119; Fax: 919.684.8591; E-mail: cdkontos@duke.edu.

1. Vanderbeek BL, Zacks DN, Talwar N, Nan B, Musch DC, Stein JD. Racial differences in age-related macular degeneration rates in the United States: a longitudinal analysis of a managed care network. Am J Ophthalmol. 2011;152(2):273-282.

2. Hu DN, Yu GP, McCormick SA, Schneider S, Finger PT. Population-based incidence of uveal melanoma in various races and ethnic groups. Am J Ophthalmol. 2005;140(4):612-617.

3. Amrock SM, Weitzman M. Diverging racial trends in neonatal infantile hemangioma diagnoses, 1979-2006. Pediatr Dermatol. 2013; 30(4):493-494

4. Wu XC, et al. Racial and ethnic variations in incidence and survival of cutaneous melanoma in the United States, 1999-2006. J Am Acad Dermatol. 2011;65(5 suppl 1):S26-S37.

5. Adini I, et al. Melanocyte-secreted fibromodulin promotes an angiogenic microenvironment. J Clin Invest. 2014;124(1):425-436.

6. Iozzo RV, Schaefer L. Proteoglycans in health and disease: novel regulatory signaling mechanisms evoked by the small leucine-rich proteoglycans. FEBS J. 2010;277(19):3864-3875.

7. Schonherr E, et al. Decorin deficiency leads to impaired angiogenesis in injured mouse cornea. J Vasc Res. 2004;41(6):499-508.

8. Soo C, et al. Differential expression of fibromodulin, a transforming growth factor-beta modulator, in fetal skin development and scarless repair. Am J Pathol. 2000;157(2):423-433.

9. Zheng Z, et al. Delayed wound closure in fibromodulin-deficient mice is associated with increased TGF- $\beta 3$ signaling. J Invest Dermatol. 2011; 131(3):769-778.

10. Jian J, et al. Fibromodulin promoted in vitro and in vivo angiogenesis. Biochem Biophys Res Commun. 
2013;436(3):530-535.

11. Rhodes JM, Simons M. The extracellular matrix and blood vessel formation: not just a scaffold J Cell Mol Med. 2007;11(2):176-205.

12. Moscatello DK, Santra M, Mann DM, McQuillan DJ, Wong AJ, Iozzo RV. Decorin suppresses tumor cell growth by activating the epidermal growth fac- tor receptor. J Clin Invest. 1998;101(2):406-412.

13. Goldoni S, et al. Decorin is a novel antagonistic ligand of the Met receptor. J Cell Biol. 2009; 185(4):743-754.

14. Schonherr E, Sunderkotter C, Iozzo RV, Schaefer L. Decorin, a novel player in the insulin-like growth factor system. J Biol Chem. 2005;280(16):15767-15772.
15. Carmeliet P, Jain RK. Molecular mechanisms and clinical applications of angiogenesis. Nature. 2011;473(7347):298-307.

16. Colin C, et al. Identification of genes differentially expressed in glioblastoma versus pilocytic astrocytoma using Suppression Subtractive Hybridization. Oncogene. 2006;25(19):2818-2826.

\title{
Aortic aneurysms in Loeys-Dietz syndrome - a tale of two pathways?
}

\author{
Frank Davis, Debra L. Rateri, and Alan Daugherty
}

Saha Cardiovascular Research Center, University of Kentucky, Lexington, Kentucky, USA.

Loeys-Dietz syndrome (LDS) is a connective tissue disorder that is characterized by skeletal abnormalities, craniofacial malformations, and a high predisposition for aortic aneurysm. In this issue of the JCI, Gallo et al. developed transgenic mouse strains harboring missense mutations in the genes encoding type I or II TGF- $\beta$ receptors. These mice exhibited several LDS-associated phenotypes. Despite being functionally defective, the mutated receptors enhanced TGF- $\beta$ signaling in vivo, inferred by detection of increased levels of phosphorylated Smad2. Aortic aneurysms in these LDS mice were ablated by treatment with the Ang II type 1 (AT1) receptor antagonist losartan. The results from this study will foster further interest into the potential therapeutic implications of AT1 receptor antagonists.

\section{TGF- $\beta$ and Ang II pathways in} thoracic aortic aneurysmal formation Aneurysms that present in the thoracic aorta have a wide range of syndromic and nonsyndromic associations (1). Marfan syndrome is one of the most researched syndromic associations and is attributed to a wide spectrum of mutations in fibrillin-1, which have been proposed to enhance the bioavailability of TGF- $\beta$ (2). The understanding of the role of TGF- $\beta$ in the etiology of thoracic aortic aneurysms (TAAs) moved forward with the development of mice that expressed the $\mathrm{C} 1039 \mathrm{G}$ mutant of fibrillin-1. These mice, colloquially referred to the "Marfan mouse," exhibit many Marfan-associated phenotypes, including a predisposition for aortic aneurysms. TGF- $\beta$-neutralizing antibody administration to Marfan mice prevented the characteristic media elastin disruption and aortic root expansion (3). Furthermore, the neutralizing antibody decreased canonical TGF- $\beta$ signaling in aortic smooth muscle cells, as defined by immunostaining of tissues for the phosphorylated form of Smad2

Conflict of interest: The authors have declared that no conflict of interest exists.

Citation for this article: J Clin Invest. 2014; 124(1):79-81. doi:10.1172/JCI73906.
(pSmad2). Another seminal discovery in the Marfan mouse was that administration of losartan, the initial member of the Ang II type 1 receptor (AT1R) blocker (ARB) class, ablated ascending aortic dilation. Subsequent studies in this mouse model have demonstrated that losartan-associated reductions in ascending aortic expansion are attributable to inhibition of the ERK pathway $(4,5)$. These groundbreaking studies in mice have assisted in development of multiple clinical trials that are evaluating efficacy of AT1R antagonism in thoracic aortic dilation of patients with Marfan syndrome (6). Although these studies shed light on the interactions between TGF- $\beta$ and AT1R signaling in TAA development, the specific mechanism of these interactions has not been elucidated (7).

TGF- $\beta$ was further implicated in the development of aortic aneurysms following the discovery of mutations in the genes encoding TGF- $\beta$ receptors in individuals afflicted with a clinical syndrome that has similarities to Marfan syndrome. This condition was subsequently termed Loeys-Dietz syndrome (LDS). Patients afflicted with LDS have a more aggressive form of ascending aortic dilation compared with those with Marfan syndrome (8). Dilation of the aortic root is detected very early, with documented aortic dissections occurring in patients with LDS as young as 3 months of age (9). Unlike Marfan syndrome, the vascular pathologies associated with LDS are more diffuse in location, as these aneurysms occur in other aortic regions and several vascular beds (10). The genetic basis of LDS is the presence of mutations in the genes encoding either type I or type II TGF- $\beta$ receptors (11). TGF- $\beta$ receptors function as multimers of both subtypes; therefore, clinical presentations are similar when defects are present in either receptor subtype. Although the TGF- $\beta$ receptor mutations result in impaired function, detection of enhanced Smad2 or Smad3 phosphorylation in surgical samples implies that TGF- $\beta$ signaling is actually increased in patients with LDS (11). The involvement of TGF- $\beta$ signaling in LDS development parallels the mechanisms of TAAs in Marfan syndrome. Unlike Marfan syndrome, there is a paucity of information on a role for Ang II in LDS.

\section{TGF- $\beta$ receptor mutations promote aortic aneurysms in LDS mouse models}

In this issue of the JCI, Gallo et al. (12) generated an array of mouse models with TGF- $\beta$ receptor dysfunction. These mouse models included mice with haploinsufficiency of either TGF- $\beta$ receptor (Tgfbr $1^{+/-}$ or Tgfbr2 ${ }^{+/-}$mice), knockin of LDS-associated alleles (Tgfbr $1^{M 318 R}$ or Tgfbr2 $2^{\text {G357W }}$ mice), and transgenic overexpression of the Tgfbr $2^{\text {G357W }}$ mutant. Haploinsufficiency of either receptor subtype did not produce vascular pathologies; however, heterogenous knockin of Tgfbr $1^{M 318 R}$ or Tgfbr ${ }^{\mathrm{G} 357 \mathrm{~W}}$ mutations or transgenic overexpression of mutated Tgfbr $2^{\text {G357W }}$ led to severe aortic pathologies. These included 